\title{
Charge and spin photocurrents in the Rashba model
}

\author{
Frank Freimuth ${ }^{1,2}$ * Stefan Blügel ${ }^{1}$, and Yuriy Mokrousov ${ }^{1,2}$ \\ ${ }^{1}$ Peter Grünberg Institut and Institute for Advanced Simulation, \\ Forschungszentrum Jülich and JARA, 52425 Jülich, Germany and \\ ${ }^{2}$ Institute of Physics, Johannes Gutenberg University Mainz, 55099 Mainz, Germany
}

(Dated: February 19, 2021)

\begin{abstract}
In metallic noncentrosymmetric crystals and at surfaces the response of spin currents and charge currents to applied electric fields contains contributions that are second order in the electric field, which are forbidden by symmetry in centrosymmetric systems. Thereby, photocurrents and spin photocurrents can be generated in inversion asymmetric metals by the application of femtosecond laser pulses. We study the laser-induced charge current in the ferromagnetic Rashba model with inplane magnetization and find that this magnetic photogalvanic effect can be tuned to be comparable in size to the laser-induced photocurrents measured experimentally in magnetic bilayer systems such as $\mathrm{Co} / \mathrm{Pt}$. Additionally, we show that femtosecond laser pulses excite strong spin currents in the nonmagnetic Rashba model when the Rashba parameter is large.
\end{abstract}

\section{INTRODUCTION}

The generation of in-plane charge currents by application of ultrashort laser pulses to magnetic bilayer systems with structural inversion asymmetry - such as $\mathrm{Co} / \mathrm{Pt}$, $\mathrm{Co} / \mathrm{Ta}$ and $\mathrm{Co}_{20} \mathrm{Fe}_{60} \mathrm{~B}_{20} / \mathrm{W}$ - is currently attracting attention, because it paves the way to ultrafast electronics, because the resulting terahertz $(\mathrm{THz})$ signals can be used to develop efficient table-top THz-emitters [1, 2], and because these charge currents contain information about several important effects, such as superdiffusive spin-currents [3, 4], spin Hall angles, the inverse Faraday effect (IFE), and the inverse spin-orbit torque (SOT) [5]. So far, two mechanisms for in-plane photocurrent generation in magnetic bilayers have been identified in experiments: First, the laser pulse triggers a superdiffusive spin current [6 9], which flows from the magnetic into the nonmagnetic layer and which is converted into an in-plane electric current by the inverse spin-Hall effect [1, 3]. Second, the IFE can be used to induce magnetization dynamics in the ferromagnetic layer [10], which drives an in-plane electric current due to the inverse SOT [5, 11]. Additionally, it has been shown theoretically that electric currents are generated if the exchange splitting varies in time after laser excitation [12]. Thus, the laser-induced photocurrents contain also information about whether ultrafast demagnetization is dominated by an exchange-field collapse [13] or by the excitation of transverse spin fluctuations [14]. Therefore, they can be used to study the nature of ultrafast demagnetization, as an alternative or complementary tool to conductivity measurements [15] and photoelectron spectroscopy [16].

Circularly polarized light induces an electric current even in noncentrosymmetric nonmagnetic semiconductors, which is known as the circular photogalvanic effect $[17-19]$. The question therefore arises whether in noncentrosymmetric magnetic metals there exists an

\footnotetext{
* Corresp. author: f.freimuth@fz-juelich.de
}

effect similar to the circular photogalvanic effect and whether such an effect contributes to the laser-induced charge currents in magnetic bilayer systems [20]. Effects from the interfacial spin-orbit interaction (SOI) in magnetic bilayer systems can be studied based on the Rashba model 21]. In the nonmagnetic Rashba model light can induce out-of-plane charge currents only and no in-plane charge currents due to symmetry. However, the magnetization vector in magnetic bilayers lowers the symmetry and one may thus expect additional electric currents perpendicular to the light wave vector, i.e., in-plane charge currents when the light wave vector is perpendicular to the bilayer interface and when the magnetization is inplane. This effect can be considered as the magnetic photogalvanic effect.

Also pure spin currents can be excited by light in noncentrosymmetric nonmagnetic semiconductors 22 24], in graphene [25] deposited on a substrate or subject to an external out-of-plane electric field, and in organicinorganic halide $\mathrm{CH}_{3} \mathrm{NH}_{3} \mathrm{PbI}_{3}$ [26], which is an important step towards ultrafast spintronics. Since a very strong Rashba effect has been found in $\mathrm{Bi} / \mathrm{Ag}(111)$ surface alloys [27], one may expect very efficient generation of spin photocurrents in this metallic surface, which would make $\mathrm{Bi} / \mathrm{Ag}(111)$ attractive for ultrafast metallic spintronics applications.

In this work we study the laser-induced in-plane charge currents in the ferromagnetic Rashba model in order to find out how large photocurrents can be that are generated directly by the interfacial Rashba SOI in magnetic bilayer systems without involving the generation of superdiffusive spin-currents or the excitation of magnetization dynamics through the IFE. Thereby we extend the list of suggested mechanisms for the generation of inplane photocurrents by light in magnetic bilayer systems. In view of the discovery of more and more nonmagnetic systems with a giant Rashba effect 27-32] we investigate also the laser-induced pure spin current in the nonmagnetic Rashba model for large SOI strength, in order to show that very strong spin currents can be generated optically in such materials. 
This paper is organized as follows. In Sec. II we describe the formalism that we use to compute the laserinduced charge currents and spin currents, which is based on the Keldysh nonequilibrium formalism. In Sec. III we discuss based on symmetry arguments which components of the laser-induced charge currents and spin currents can exist and which components are zero in the Rashba model. In Sec. IVA we present numerical results for the laser-induced charge current in the ferromagnetic Rashba model and in Sec. IVB we discuss the numerical results for the laser-induced spin current in the nonmagnetic Rashba model. This paper ends with a summary in Sec. V.

\section{FORMALISM}

In experiments femtosecond laser pulses are used very often instead of continuous laser beams, because thereby much larger electric field strengths can be applied. Additionally, a novel type of $\mathrm{THz}$ emitter uses excitation by femtosecond laser pulses [1, 2]. We assume that the response to laser pulses can be modelled by considering the time-dependent intensity $I(t)$ of the laser pulse and by assuming that the response at time $t$ agrees with the hypothetical response to a continuous laser beam with constant intensity $I$ given by $I=I(t)$. This approximation is justified for the parameters used in this work, because 50 -fs pulses of light wavelength $800 \mathrm{~nm}$ correspond to roughly 20 oscillations of the electric field vector. Moreover, this approximation has predicted the amplitudes of the effects satisfactorily [33].

\section{A. Laser-induced charge current}

The response that arises at the second order in the perturbing electric field of a continuous laser beam with frequency $\omega$ contains a dc contribution and an ac contribution with frequency $2 \omega$. Here, we are only interested in the dc contribution. The dc electric current response to a continuous laser beam appears as a $\mathrm{THz}$ electric current pulse when femtosecond laser pulses are used instead of a continuous laser beam. Therefore, in the following we discuss the expressions to compute the dc electric current driven by a continuous laser beam with light frequency $\omega$.

To derive expressions suitable to describe the laserinduced electric current one can proceed in close analogy to the case of torques that arise at the second order in the perturbing electric field of the laser, which were discussed in detail in Ref. 33. We do not present the detailed derivation here but only the final expression. The dc electric current density that arises at second order in the electric field of a continuous laser beam of frequency $\omega$ can be written as

$$
J_{\alpha}=\frac{a_{0}^{2} e I}{\hbar c}\left(\frac{\mathcal{E}_{\mathrm{H}}}{\hbar \omega}\right)^{2} \operatorname{Im} \sum_{\beta \gamma} \epsilon_{\beta} \epsilon_{\gamma}^{*} \varphi_{\alpha \beta \gamma},
$$

where $\varphi_{\alpha \beta \gamma}=\chi_{\beta \gamma}^{v_{\alpha}}$ and the response of a general observable $\mathcal{O}$ to monochromatic light of frequency $\omega$ is given by

$$
\begin{aligned}
\chi_{\beta \gamma}^{\mathcal{O}}= & \frac{2}{a_{0} \mathcal{E}_{\mathrm{H}}} \int \frac{\mathrm{d}^{2} k}{(2 \pi)^{2}} \int \mathrm{d} \mathcal{E} \operatorname{Tr}[ \\
& f(\mathcal{E}) \mathcal{O} G_{\boldsymbol{k}}^{\mathrm{R}}(\mathcal{E}) v_{\beta} G_{\boldsymbol{k}}^{\mathrm{R}}(\mathcal{E}-\hbar \omega) v_{\gamma} G_{\boldsymbol{k}}^{\mathrm{R}}(\mathcal{E}) \\
- & f(\mathcal{E}) \mathcal{O} G_{\boldsymbol{k}}^{\mathrm{R}}(\mathcal{E}) v_{\beta} G_{\boldsymbol{k}}^{\mathrm{R}}(\mathcal{E}-\hbar \omega) v_{\gamma} G_{\boldsymbol{k}}^{\mathrm{A}}(\mathcal{E}) \\
+ & f(\mathcal{E}) \mathcal{O} G_{\boldsymbol{k}}^{\mathrm{R}}(\mathcal{E}) v_{\gamma} G_{\boldsymbol{k}}^{\mathrm{R}}(\mathcal{E}+\hbar \omega) v_{\beta} G_{\boldsymbol{k}}^{\mathrm{R}}(\mathcal{E}) \\
- & f(\mathcal{E}) \mathcal{O} G_{\boldsymbol{k}}^{\mathrm{R}}(\mathcal{E}) v_{\gamma} G_{\boldsymbol{k}}^{\mathrm{R}}(\mathcal{E}+\hbar \omega) v_{\beta} G_{\boldsymbol{k}}^{\mathrm{A}}(\mathcal{E}) \\
+ & f(\mathcal{E}-\hbar \omega) \mathcal{O} G_{\boldsymbol{k}}^{\mathrm{R}}(\mathcal{E}) v_{\beta} G_{\boldsymbol{k}}^{\mathrm{R}}(\mathcal{E}-\hbar \omega) v_{\gamma} G_{\boldsymbol{k}}^{\mathrm{A}}(\mathcal{E}) \\
+ & \left.f(\mathcal{E}+\hbar \omega) \mathcal{O} G_{\boldsymbol{k}}^{\mathrm{R}}(\mathcal{E}) v_{\gamma} G_{\boldsymbol{k}}^{\mathrm{R}}(\mathcal{E}+\hbar \omega) v_{\beta} G_{\boldsymbol{k}}^{\mathrm{A}}(\mathcal{E})\right] .
\end{aligned}
$$

Here, $a_{0}=4 \pi \epsilon_{0} \hbar^{2} /\left(m e^{2}\right)$ is Bohr's radius, $I$ is the intensity of light, $c$ is the velocity of light, $\mathcal{E}_{\mathrm{H}}=e^{2} /\left(4 \pi \epsilon_{0} a_{0}\right)$ is the Hartree energy and $f(\mathcal{E})$ is the Fermi distribution function. $v_{\beta}$ is the $\beta$ th component of the velocity operator, $e$ is the elementary positive charge, $\mathcal{E}_{\mathrm{F}}$ is the Fermi energy,

$$
G_{\boldsymbol{k}}^{\mathrm{R}}(\mathcal{E})=\hbar \sum_{n} \frac{|\boldsymbol{k} n\rangle\langle\boldsymbol{k} n|}{\mathcal{E}-\mathcal{E}_{\boldsymbol{k} n}+i \Gamma}
$$

is the retarded Green function and $G_{\boldsymbol{k}}^{\mathrm{A}}(\mathcal{E})=\left[G_{\boldsymbol{k}}^{\mathrm{R}}(\mathcal{E})\right]^{\dagger}$ is the advanced Green function. The energy of the state $|\boldsymbol{k} n\rangle$ of an electron in band $n$ at $k$-point $\boldsymbol{k}$ is $\mathcal{E}_{\boldsymbol{k} n}$. The parameter $\Gamma$ describes the lifetime broadening of the electronic states. $\epsilon_{\beta}$ is the $\beta$ th component of the polarization vector of the light. Circularly polarized light with light wave vector along the $z$ direction is described by $\boldsymbol{\epsilon}=(1, \lambda i, 0) / \sqrt{2}$, where $\lambda= \pm 1$ controls the light helicity. In order to obtain the coefficients $\varphi_{\alpha \beta \gamma}=\chi_{\beta \gamma}^{v_{\alpha}}$ in Eq. (11) one only needs to substitute $\mathcal{O}$ in Eq. (2) by $v_{\alpha}$, i.e., by the $\alpha$ th component of the velocity operator. Note that while the Green's function is chosen to be band-diagonal in Eq. (3) the velocity operator and $\mathcal{O}$ are not band-diagonal and induce interband transitions in Eq. (2).

\section{B. Laser-induced spin current}

Similarly, the dc spin-current density that arises in second order response to the electric field of the laser can be written as

$$
J_{\alpha}^{s}=-\frac{a_{0}^{2} I}{4 c}\left(\frac{\mathcal{E}_{\mathrm{H}}}{\hbar \omega}\right)^{2} \operatorname{Im} \sum_{j k} \epsilon_{\beta} \epsilon_{\gamma}^{*} \phi_{\alpha \beta \gamma}^{s},
$$

where $\phi_{\alpha \beta \gamma}^{s}=\chi_{\beta \gamma}^{\left\{v_{\alpha}, \sigma_{s}\right\}}$, i.e., $\phi_{\alpha \beta \gamma}^{s}$ is obtained from $\chi_{\beta \gamma}^{\mathcal{O}}$ in Eq. (2) by the substitution $\mathcal{O}=\left\{v_{\alpha}, \sigma_{s}\right\}$. Here, $\sigma_{s}$ 
$(s=x, y, z)$ are the Pauli spin-matrices and $J_{\alpha}^{s}$ is the component of the spin-current density where the spin of the carriers is oriented in $s$ direction and the carriers move along the $\alpha$ direction.

\section{Rashba model}

We investigate the laser-induced charge current and spin current in the Rashba model (see Ref. 21] for a recent review on the Rashba model). The Rashba model with an additional exchange splitting is given by

$$
H^{\mathrm{R}}=\frac{-\hbar^{2}}{2 m_{e}} \Delta-i \alpha^{\mathrm{R}}\left(\boldsymbol{\nabla} \times \hat{\boldsymbol{e}}_{z}\right) \cdot \boldsymbol{\sigma}+\frac{\Delta V}{2} \boldsymbol{\sigma} \cdot \hat{\boldsymbol{n}}(\boldsymbol{r}),
$$

where the first, second and third terms on the righthand side describe the kinetic energy, the Rashba SOI and the exchange interaction, respectively. By modelling the experimentally measured DzyaloshinskiiMoriya interaction the Rashba parameter in $\mathrm{Co} / \mathrm{Pt}$ bilayers was estimated to be $\alpha^{\mathrm{R}}=0.095 \mathrm{eV} \AA$ 34]. The same order of magnitude of $\alpha^{\mathrm{R}}$ was estimated for $\mathrm{Ni}_{80} \mathrm{Fe}_{20} / \mathrm{Pt}$ [35] Substantially larger values of $\alpha^{\mathrm{R}}$ have been reported for $\mathrm{Bi} / \mathrm{Ag}(111)$ surface alloys $\left(\alpha^{\mathrm{R}}=\right.$ $3.05 \mathrm{eV} \AA[27])$, for BiTeI $\left(\alpha^{\mathrm{R}}=3.85 \mathrm{eV} \AA[29]\right)$, and for $\mathrm{Pb}_{1-x} \mathrm{Sn}_{x} \mathrm{Te}\left(\alpha^{\mathrm{R}}=3.8 \mathrm{eV} \AA[32]\right)$.

In the Rashba model the velocity operator $\boldsymbol{v}_{\boldsymbol{k}}=$ $e^{-i \boldsymbol{k} \cdot \boldsymbol{r}} \boldsymbol{v} e^{i \boldsymbol{k} \cdot \boldsymbol{r}}$ is given by

$$
\boldsymbol{v}_{\boldsymbol{k}}=\frac{1}{\hbar} \frac{\partial H^{\mathrm{R}}}{\partial \boldsymbol{k}}=\frac{\hbar}{m} \boldsymbol{k}+\alpha^{\mathrm{R}} \hat{\boldsymbol{e}}_{z} \times \boldsymbol{\sigma}
$$

Due to the term $\alpha^{\mathrm{R}} \hat{\boldsymbol{e}}_{z} \times \boldsymbol{\sigma}$ the velocity operator $\boldsymbol{v}_{\boldsymbol{k}}$ does not commute with the Hamiltonian $H^{\mathrm{R}}$. Consequently, the eigenstates of the Hamiltonian $H^{\mathrm{R}}$ are not simultaneously eigenstates of the velocity operator $\boldsymbol{v}_{\boldsymbol{k}}$. When the Green's function is chosen to be band-diagonal like in Eq. (3) the velocity operator is not band-diagonal and induces interband transitions in Eq. (2).

\section{SYMMETRY PROPERTIES}

The response of the charge current to the second-order perturbation by an applied electric field is described by a polar tensor of third rank. Therefore, it is nonzero only in noncentrosymmetric crystals. Similarly, the response of the spin-current to the second order perturbation by an applied electric field, which is described by an axial tensor of fourth rank, is nonzero only in noncentrosymmetric crystals [36]. In the following we discuss which components of the laser-induced charge current and of the laser-induced spin current are allowed by symmetry in the Rashba model.

\section{A. Laser-induced charge current}

Circularly polarized light with wave vector parallel to the $z$ direction does not induce in-plane charge currents in the nonmagnetic Rashba model due to the rotational symmetry around the $z$ axis: $c_{2}$ rotation flips both $J_{x}$ and $J_{y}$ but does not change the helicity $\lambda$ (see Table $\llbracket$ ). Similarly, linearly polarized light does not induce in-plane charge currents in this case.

TABLE I. Effect of $c_{2}$ rotation around the $z$ axis, effect of mirror reflection $\mathcal{M}_{z x}$ at the $z x$ plane, and effect of mirror reflection $\mathcal{M}_{y z}$ at the $y z$ plane on light helicity $\lambda$, magnetization $\boldsymbol{M}$, current density $\boldsymbol{J}$, and spin current density $J_{\alpha}^{s}$. The magnetization $\boldsymbol{M}$ transforms like an axial vector, while the current density $\boldsymbol{J}$ transforms like a polar vector.

\begin{tabular}{c|c|c|c|c|c|c|c|c|c|c}
\hline \hline & $\lambda$ & $M_{y}$ & $J_{x}$ & $J_{y}$ & $J_{x}^{y}$ & $J_{y}^{x}$ & $J_{x}^{x}$ & $J_{y}^{y}$ & $J_{x}^{z}$ & $J_{y}^{z}$ \\
\hline$c_{2}$ & $\lambda$ & $-M_{y}$ & $-J_{x}$ & $-J_{y}$ & $J_{x}^{y}$ & $J_{y}^{x}$ & $J_{x}^{x}$ & $J_{y}^{y}$ & $-J_{x}^{z}$ & $-J_{y}^{z}$ \\
\hline $\mathcal{M}_{z x}$ & $-\lambda$ & $M_{y}$ & $J_{x}$ & $-J_{y}$ & $J_{x}^{y}$ & $J_{y}^{x}$ & $-J_{x}^{x}$ & $-J_{y}^{y}$ & $-J_{x}^{z}$ & $J_{y}^{z}$ \\
\hline $\mathcal{M}_{y z}$ & $-\lambda$ & $-M_{y}$ & $-J_{x}$ & $J_{y}$ & $J_{x}^{y}$ & $J_{y}^{x}$ & $-J_{x}^{x}$ & $-J_{y}^{y}$ & $J_{x}^{z}$ & $-J_{y}^{z}$ \\
\hline \hline
\end{tabular}

In the ferromagnetic Rashba model with magnetization along $y$ light polarized linearly along $x$ or $y$ induces a current density $J_{x}$ that is odd in magnetization, because $\mathcal{M}_{y z}$ (or alternatively $c_{2}$ ) flips the magnetization (axial vector) and $J_{x} . J_{y}$ is not allowed by symmetry due to $\mathcal{M}_{z x}$, which leaves the magnetization invariant but flips $J_{y}$.

In the ferromagnetic Rashba model with magnetization along $y$ circularly polarized light induces $J_{y}$, which is odd in the helicity of light and odd in magnetization, because $\mathcal{M}_{y z}$ flips the light helicity and the magnetization but preserves $J_{y}$, while $\mathcal{M}_{z x}$ preserves the magnetization, but flips the light helicity and $J_{y}$. In this case symmetry allows also a nonzero $J_{x}$, which is even in the helicity of light and odd in magnetization, because $\mathcal{M}_{z x}$ flips the light helicity and preserves $J_{x}$ and the magnetization, while $\mathcal{M}_{y z}$ flips the light helicity, the magnetization and $J_{x}$.

These symmetry properties of the magnetic photogalvanic effect in the ferromagnetic Rashba model are summarized in Table $\amalg$.

TABLE II. Symmetry properties of the magnetic photogalvanic effect in the ferromagnetic Rashba model with magnetization parallel to the $y$ axis. $\emptyset$ means no effect. $M_{y}$ means odd in magnetization, i.e., the effect changes sign when the magnetization is antiparallel to the $y$ axis. $\lambda M_{y}$ means odd in the light helicity and odd in the magnetization. $|\lambda| M_{y}$ means even in the light helicity and odd in the magnetization.

\begin{tabular}{c|c|c|}
\hline \hline & circularly polarized & linearly polarized $(\boldsymbol{\epsilon} \| x$ or $\boldsymbol{\epsilon} \| y)$ \\
\hline$J_{x}$ & $|\lambda| M_{y}$ & $M_{y}$ \\
\hline$J_{y}$ & $\lambda M_{y}$ & $\emptyset$ \\
\hline \hline
\end{tabular}




\section{B. Laser-induced spin current}

Nonmagnetic Rashba model

We first discuss the symmetry properties of laserinduced spin currents in the nonmagnetic Rashba model.

For light polarized linearly along $x$ the spin-current density $J_{x}^{y}$ is allowed by symmetry: $\mathcal{M}_{z x}$ does not flip $J_{x}^{y}$. $\mathcal{M}_{y z}$ does not flip $J_{x}^{y}$ either, because it flips both the velocity of the carriers (polar vector) and their spin (axial vector). In this case also $J_{y}^{x}$ is allowed by symmetry: $\mathcal{M}_{y z}$ does not flip $J_{y}^{x} \cdot \mathcal{M}_{z x}$ does not flip $J_{y}^{x}$ either, because it flips both the velocity of the carriers and their spin. However, $J_{x}^{x}$ is forbidden by symmetry in this case, because $\mathcal{M}_{z x}$ flips only the spin of the carriers and not their velocity and therefore it flips $J_{x}^{x}$. Similarly, $J_{y}^{y}$ is forbidden by symmetry in this case, because $\mathcal{M}_{y z}$ flips $J_{y}^{y}$. Finally, also $J_{x}^{z}$ and $J_{y}^{z}$ are forbidden by symmetry in this case, because $\mathcal{M}_{z x}$ flips $J_{x}^{z}$, and $\mathcal{M}_{y z}$ flips $J_{y}^{z}$.

For circularly polarized light $J_{x}^{y}$ and $J_{y}^{x}$ are allowed by symmetry, if they are even in the helicity of light, because both $\mathcal{M}_{z x}$ and $\mathcal{M}_{y z}$ flip the helicity of the light. Since $\mathcal{M}_{z x}$ flips $J_{x}^{z}$ but $\mathcal{M}_{y z}$ does not, $J_{x}^{z}$ is forbidden by symmetry. Similarly, $J_{y}^{z}$ is forbidden by symmetry. Both $\mathcal{M}_{z x}$ and $\mathcal{M}_{y z}$ flip $J_{x}^{x}$ and $J_{y}^{y}$. Therefore, $J_{x}^{x}$ and $J_{y}^{y}$ are allowed by symmetry, if they are odd in the helicity of light.

The symmetry properties of the laser-induced spin current in the nonmagnetic Rashba model are summarized in Table III ( \{\} -brackets in the Table).

\section{Ferromagnetic Rashba model}

Next, we discuss the symmetry properties of the laserinduced spin currents in the ferromagnetic Rashba model with magnetization along $y$.

Linearly polarized light with polarization along $x: \mathcal{M}_{z x}$ does not flip $J_{x}^{y}$ and preserves the magnetization. $\mathcal{M}_{y z}$ flips the magnetization, but it does not flip $J_{x}^{y}$ because it flips both the carrier velocity and the spin. Thus, $J_{x}^{y}$ is even in magnetization. $\mathcal{M}_{z x}$ does not flip $J_{y}^{x}$, because it flips both the carrier velocity and the spin. It also preserves the magnetization. $\mathcal{M}_{y z}$ does not flip $J_{y}^{x}$, but it flips the magnetization. Thus, $J_{y}^{x}$ is even in magnetization. $J_{x}^{x}$ is forbidden by symmetry, because $\mathcal{M}_{z x}$ flips $J_{x}^{x}$ but preserves the magnetization. Similarly, $J_{y}^{y}$ is forbidden by symmetry, because $\mathcal{M}_{z x}$ flips $J_{y}^{y}$ and preserves the magnetization. Also $J_{x}^{z}$ is forbidden by symmetry, because $\mathcal{M}_{z x}$ flips $J_{x}^{z}$ and preserves the magnetization. $\mathcal{M}_{z x}$ preserves $J_{y}^{z}$ and the magnetization, while the $y z$ mirror plane flips $J_{y}^{z}$ and the magnetization. Consequently, $J_{y}^{z}$ is allowed by symmetry and it is odd in the magnetization.

Circularly polarized light: Both $\mathcal{M}_{z x}$ and $\mathcal{M}_{y z}$ flip the helicity of the light. Thus, $J_{x}^{y}$ and $J_{y}^{x}$ are allowed by symmetry, if they are even in the helicity of the light. Since $\mathcal{M}_{y z}$ flips the magnetization while $\mathcal{M}_{z x}$ preserves it, $J_{x}^{y}$ and $J_{y}^{x}$ are even in the magnetization. $\mathcal{M}_{z x}$ flips $J_{x}^{z}$, flips the helicity and preserves the magnetization. $\mathcal{M}_{y z}$ preserves $J_{x}^{z}$, flips the helicity and flips the magnetization. The combination of $\mathcal{M}_{z x}$ and $\mathcal{M}_{y z}$ flips $J_{x}^{z}$, flips the magnetization and preserves the helicity. Thus, $J_{x}^{z}$ is odd in the magnetization and odd in the helicity. $\mathcal{M}_{z x}$ preserves $J_{y}^{z}$, flips the helicity and preserves the magnetization. $\mathcal{M}_{y z}$ flips $J_{y}^{z}$, flips the helicity and flips the magnetization. The combination of $\mathcal{M}_{z x}$ and $\mathcal{M}_{y z}$ flips $J_{y}^{z}$, flips the magnetization and preserves the helicity. Thus, $J_{y}^{z}$ is odd in the magnetization and even in the helicity. Both $\mathcal{M}_{z x}$ and $\mathcal{M}_{y z}$ flip $J_{x}^{x}$ and $J_{y}^{y}$. Therefore, $J_{x}^{x}$ and $J_{y}^{y}$ are odd in the helicity of light. Since $\mathcal{M}_{y z}$ flips the magnetization while $\mathcal{M}_{z x}$ preserves it, $J_{x}^{x}$ and $J_{y}^{y}$ are even in the magnetization.

The symmetry properties of the laser-induced spin current in the magnetic Rashba model with magnetization in $y$ direction are summarized in Table III)([]-brackets in the Table).

TABLE III. Symmetry properties of the laser-induced spincurrent density in the nonmagnetic Rashba model (shown in \{\} -brackets) and of the ferromagnetic Rashba model with magnetization parallel to the $y$ axis (shown in []-brackets). $\emptyset$ indicates that there is no effect, while $\checkmark$ signals that there is one. $M_{y}$ means that the effect is odd in the magnetization, i.e., it changes sign when the magnetization is antiparallel to the $y$ axis. $\left|M_{y}\right|$ signals that the effect is even in the magnetization. Effects that are even and odd in the helicity of light are indicated by $|\lambda|$ and $\lambda$, respectively.

\begin{tabular}{c|c|c|}
\hline \hline & circularly polarized & linearly polarized $(\boldsymbol{\epsilon} \| x$ or $\boldsymbol{\epsilon} \| y)$ \\
\hline$J_{x}^{x}$ & $\{\lambda\},\left[\lambda\left|M_{y}\right|\right]$ & $\{\emptyset\},[\emptyset]$ \\
\hline$J_{x}^{y}$ & $\{|\lambda|\},\left[|\lambda|\left|M_{y}\right|\right]$ & $\{\checkmark\},\left[\left|M_{y}\right|\right]$ \\
\hline$J_{x}^{z}$ & $\{\emptyset\},\left[\lambda M_{y}\right]$ & $\{\emptyset\},[\emptyset]$ \\
\hline$J_{y}^{x}$ & $\{|\lambda|\},\left[|\lambda|\left|M_{y}\right|\right]$ & $\{\checkmark\},\left[\left|M_{y}\right|\right]$ \\
\hline$J_{y}^{y}$ & $\{\lambda\},\left[\lambda\left|M_{y}\right|\right]$ & $\{\emptyset\},[\emptyset]$ \\
\hline$J_{y}^{z}$ & $\{\emptyset\},\left[M_{y}|\lambda|\right]$ & $\{\emptyset\},\left[M_{y}\right]$ \\
\hline \hline
\end{tabular}

\section{RESULTS}

In the following we present results for the chargecurrent density and for the spin-current density induced by a continuous laser beam, which we calculate from Eq. (11) and Eq. (4), respectively. In all results presented below, we assume that the intensity is given by $I=$ $10 \mathrm{GW} / \mathrm{cm}^{2}$ and the photon energy is set to $\hbar \omega=1.55 \mathrm{eV}$. This photon energy corresponds to $800 \mathrm{~nm}$, which is often used in experiments on $\mathrm{Co} / \mathrm{Pt}$ bilayers $1,3,3,5,20]$. The intensity $I=10 \mathrm{GW} / \mathrm{cm}^{2}$ is of the same order of magnitude as the one used in these experiments.

While the Rashba model has been used successfully to model spintronic properties qualitatively [21, 34], precise quantitative predictions are usually not possible due to the simplicity of this model. By construction, the Rashba model misses completely the contribution that 
arises from the superdiffusive spin-currents [3]. Also, the contribution originating from laser-induced magnetization dynamics is not considered below [5]. Therefore, in the following we aim only at qualitative results and order of magnitude estimates of laser-induced photocurrents in $\mathrm{Co} / \mathrm{Pt}$ bilayers that arise from photogalvanic effects. Additionally, we study a photogalvanic effect of spin-currents in the non-magnetic Rashba model.

\section{A. Laser-induced charge currents}

The laser-induced charge-current density as a function of Fermi energy $\mathcal{E}_{\mathrm{F}}$ is shown in Fig. [1 for the parameters $\alpha^{\mathrm{R}}=0.1 \mathrm{eV} \AA, \Gamma=25 \mathrm{meV}$ and $\Delta V=1 \mathrm{eV}$ when $\hat{\boldsymbol{n}}$ points in $y$ direction. As discussed in section $\amalg C \alpha^{\mathrm{R}}=0.1 \mathrm{eV} \AA$ is a suitable choice to model magnetic bilayer systems such as $\mathrm{Co} / \mathrm{Pt}$. Previously, we found that the broadening of $\Gamma=25 \mathrm{meV}$ is suitable to reproduce the experimentally measured SOTs in ab-initio calculations of $\mathrm{Co} / \mathrm{Pt}$ bilayers [37] and therefore we use it here as well. The laser-induced current density $J_{x}$ is even in the helicity $\lambda$ and for circularly polarized light it is much larger than for linearly polarized light with polarization along $x$. However, for linearly polarized light with polarization along $y$ the laser-induced current density $J_{x}$ is larger than the one for circularly polarized light by around a factor of 2 . The laser-induced current density $J_{y}$ is odd in the helicity $\lambda$ and therefore it vanishes for linearly polarized light (not shown in the figure). The finding that $J_{x}$ is even in $\lambda$ while $J_{y}$ is odd in $\lambda$ is consistent with the symmetry analysis in section IIIA

$J_{\alpha}$ in Fig. 1 starts to be nonzero roughly at $-1 \mathrm{eV}$ and it displays kinks roughly at 1eV. In Ref. [33] in Eq. (A12) we have shown that the Fermi surface contribution can be recast as a sum of two terms that contain the factors $[f(\mathcal{E}-\hbar \omega)-f(\mathcal{E})]$ and $f[(\mathcal{E}+\hbar \omega)-f(\mathcal{E})]$, respectively. The first factor is nonzero for $\mathcal{E}_{\mathrm{F}}<\mathcal{E}<\mathcal{E}_{\mathrm{F}}+\hbar \omega$, while the second factor is nonzero for $\mathcal{E}_{\mathrm{F}}-\hbar \omega<\mathcal{E}<\mathcal{E}_{\mathrm{F}}$. $J_{\alpha}$ in Fig. 11starts to be nonzero roughly when $\mathcal{E}_{\mathrm{F}}+\hbar \omega>0.5 \mathrm{eV}$, i.e., roughly at $\mathcal{E}_{\mathrm{F}}>-1 \mathrm{eV}$, because this is when the energy $\mathcal{E}$ can reach up to the bottom of the second band at $0.5 \mathrm{eV}$ due to the first factor. The second factor is most efficient when $\mathcal{E}_{\mathrm{F}}-\hbar \omega$ reaches the bottom of the first band, which is at $-0.5 \mathrm{eV}$. This happens roughly at $\mathcal{E}_{\mathrm{F}}>1 \mathrm{eV}$. At this energy there are kinks in the $J_{\alpha}$ in Fig. 1 .

$J_{y}$ increases with Fermi energy and in the range shown in Fig. 1 it is maximally $0.78 \mathrm{~mA} / \mathrm{cm}$ for $\mathcal{E}_{\mathrm{F}}=6.8 \mathrm{eV}$. Experimentally, the amplitude of the current density $J_{y}$ has been estimated to be $5 \mathrm{~mA} / \mathrm{cm}$ when a $50 \mathrm{fs}$ laser pulse with fluence $1 \mathrm{mJcm}^{-2}$ is applied to $\mathrm{Co} / \mathrm{Pt}$ bilayers [5]. Assuming a Gaussian-shaped laser pulse we estimate the peak intensity of the pulse to be $I \approx$ $2 \sqrt{\ln (2) / \pi} \mathrm{mJcm}^{-2} /(50 \mathrm{fs}) \approx 18.8 \mathrm{GWcm}^{-2}$. The values shown in Fig. 1 have been obtained for the smaller intensity of $I=10 \mathrm{GWcm}^{-2}$, for which we expect the corresponding smaller experimental peak current density of $2.7 \mathrm{~mA} / \mathrm{cm}$, which is larger than $0.78 \mathrm{~mA} / \mathrm{cm}$ by a factor

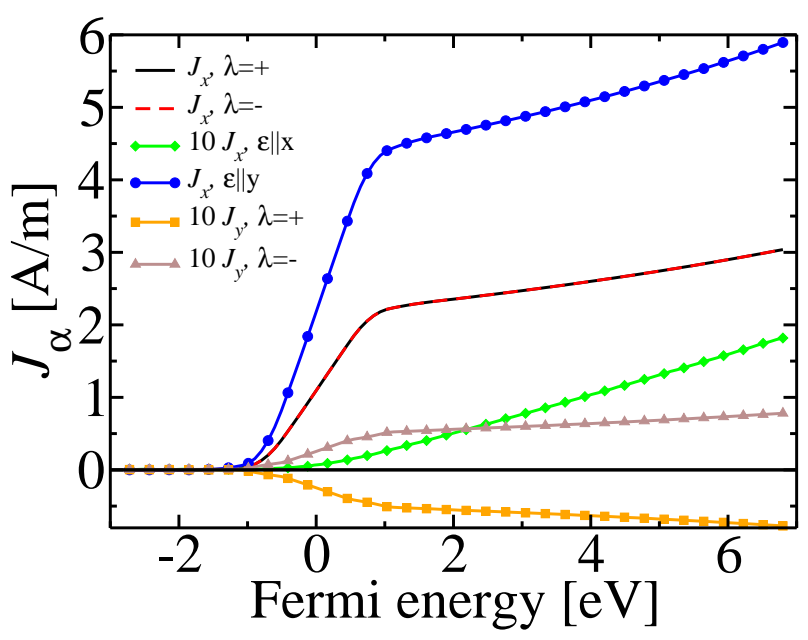

FIG. 1. Laser-induced charge-current density $J_{\alpha}$ vs. Fermi energy $\mathcal{E}_{\mathrm{F}}$ for $\hat{\boldsymbol{n}}$ in $y$ direction, $\alpha^{\mathrm{R}}=0.1 \mathrm{eV} \AA, \Delta V=1 \mathrm{eV}$, and $\Gamma=25 \mathrm{meV}$. Some curves have been multiplied by the factor 10 for better visibility, as indicated in the legend.

of 3.5. The laser-induced current density $J_{y}$ observed experimentally in $\mathrm{Co} / \mathrm{Pt}$ has been explained in terms of the IFE combined with the inverse SOT [5]. Since $0.78 \mathrm{~mA} / \mathrm{cm}$ is only smaller by a factor of 3.5 compared to the experimental value of $2.7 \mathrm{~mA} / \mathrm{cm}$ estimated for $\mathrm{Co} / \mathrm{Pt}$, we expect that this magnetic circular photogalvanic effect is in general a non-negligible contribution to $J_{y}$. If materials with small IFE or small inverse SOT are used it is likely that the contribution from the magnetic circular photogalvanic effect is dominant in $J_{y}$.

For circularly polarized light, the current density $J_{x}$ reaches $3 \mathrm{~A} / \mathrm{m}$ at $\mathcal{E}_{\mathrm{F}}=6.8 \mathrm{eV}$ in Fig. 11, which is considerably larger than $J_{y}$. Also in the experiments on $\mathrm{Co} / \mathrm{Pt} J_{x}$ is found to be much larger than $J_{y}[5]$. In the experiments, $J_{y}$ depends strongly on the Pt thickness and varies between $3.3 \mathrm{~A} / \mathrm{m}(1.3 \mathrm{~nm}$ thick $\mathrm{Pt})$ and $14.4 \mathrm{~A} / \mathrm{m}(3.9 \mathrm{~nm}$ thick $\mathrm{Pt})$ when a $50 \mathrm{fs}$ laser pulse with fluence $1 \mathrm{mJcm}^{-2}$ is used. We estimate that the corresponding current densities expected for the smaller intensity of $I=10 \mathrm{GWcm}^{-2}$ range between $1.8 \mathrm{~A} / \mathrm{m}$ and $7.7 \mathrm{~A} / \mathrm{m}$. The experimentally measured $J_{x}$ in magnetic bilayer systems has been interpreted to originate from the superdiffusive spin-current that is converted into a charge current by the inverse spin Hall effect [3]. This interpretation is supported by the very good correlation between the spin Hall conductivity of the normal metal (NM) layer and the measured $\mathrm{THz}$ amplitude in $\mathrm{Co}_{20} \mathrm{Fe}_{60} \mathrm{~B}_{20}(3 \mathrm{~nm}) / \mathrm{NM}(3 \mathrm{~nm})$ stacks [1]. Even though our theoretical values of $J_{x}$ shown in Fig. 1 describe a magnetic photogalvanic effect and do not contain the mechanism of generating a charge current by conversion of a superdiffusive spin current, the maximal value of $3 \mathrm{~A} / \mathrm{m}$ in Fig. 11 is non-negligible compared to the current density $J_{x}$ measured in $\mathrm{Co} / \mathrm{Pt}$ bilayer systems. Therefore, we expect that for suitable material combinations 


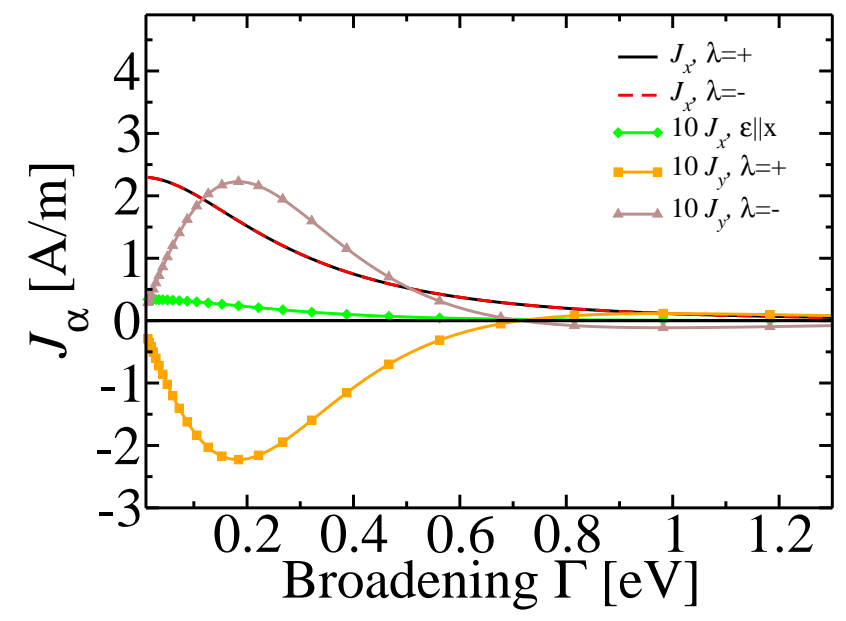

FIG. 2. Laser-induced charge-current density $J_{\alpha}$ vs. broadening $\Gamma$ for $\hat{\boldsymbol{n}}$ in $y$ direction, $\alpha^{\mathrm{R}}=0.1 \mathrm{eV} \AA, \Delta V=1 \mathrm{eV}$, and $\mathcal{E}_{\mathrm{F}}=1.36 \mathrm{eV}$. Some curves have been multiplied by the factor 10 for better visibility, as indicated in the legend.

the magnetic photogalvanic effect can compete with the conversion of superdiffusive spin current by the inverse spin Hall effect. In particular when the spin Hall conductivity of NM is small or when the NM thickness is much smaller than the hot-electron relaxation length [1] we expect significant contributions from the magnetic photogalvanic effect to the current density $J_{x}$.

It might be possible to identify the contribution of the magnetic photogalvanic effect to the current density $J_{x}$ in experiments by measuring the dependence of $J_{x}$ on the polarization of light: According to Fig. 1 the current density $J_{x}$ generated by linearly polarized light depends strongly on whether the light polarization vector is along $x$ or along $y$. On the other hand, the generation of superdiffusive spin currents is not expected to depend on the direction of the light polarization vector. Therefore, a strong dependence of $J_{x}$ on the light polarization vector is a clear indication of the magnetic photogalvanic effect.

Like the field-like contribution to the SOT [38] the magnetic photogalvanic effect is sensitive to the interfacial SOI in magnetic bilayer systems. Therefore, we expect that the magnitude of the magnetic photogalvanic effect is correlated with the magnitude of the field-like component of the SOT. On the other hand, the contribution from the conversion of superdiffusive spin current by the inverse spin Hall effect is expected to correlate with the antidamping component of the SOT. These two contributions to $J_{x}$ may therefore also be distinguished in experiments via their different dependence on the interfacial SOI. In contrast, we expect that it is more difficult to identify the contribution of the magnetic photogalvanic effect to the current density $J_{y}$ in experiments, because it competes with the current generated by the combined action of the IFE and the inverse field-like SOT, i.e., both contributions to $J_{y}$ are sensitive to the interfacial SOI.

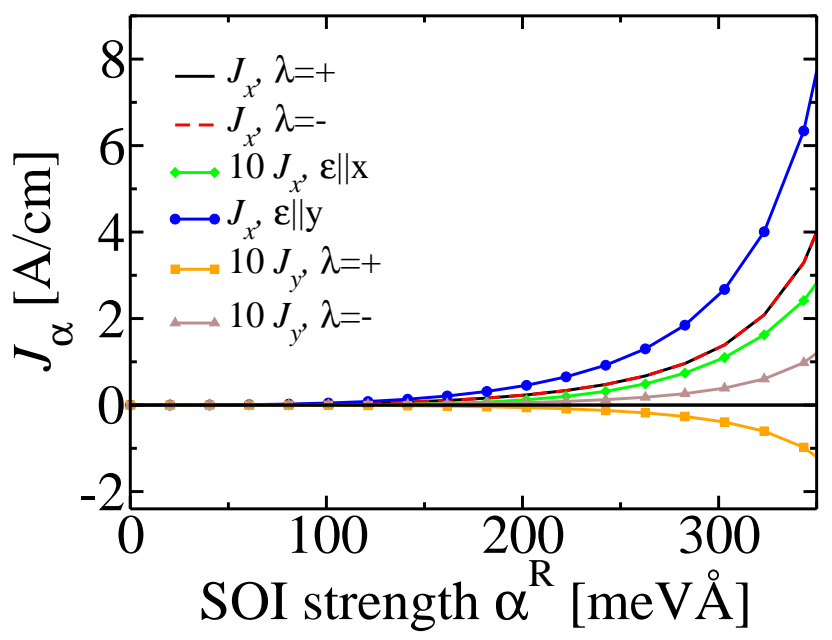

FIG. 3. Laser-induced charge-current density $J_{\alpha}$ vs. SOI strength $\alpha^{\mathrm{R}}$ for $\hat{\boldsymbol{n}}$ in $y$ direction, $\Delta V=1 \mathrm{eV}, \mathcal{E}_{\mathrm{F}}=1.36 \mathrm{eV}$, and $\Gamma=25 \mathrm{meV}$. Some curves have been multiplied by the factor 10 for better visibility, as indicated in the legend.

Next, we discuss the dependence of the magnetic photogalvanic effect on the lifetime broadening $\Gamma$. The laserinduced charge-current density is shown as a function of $\Gamma$ in Fig. 2, where we set $\mathcal{E}_{\mathrm{F}}=1.36 \mathrm{eV}, \alpha^{\mathrm{R}}=0.1 \mathrm{eV} \AA$, $\Delta V=1 \mathrm{eV}$ and $\hat{\boldsymbol{n}}$ points in $y$ direction. While the current density $J_{x}$ decreases monotonically with increasing broadening $\Gamma$ the current density $J_{y}$ exhibits a maximum at around $\Gamma=200 \mathrm{meV}$. This suggests that $J_{y}$ can be maximized in magnetic bilayer systems such as $\mathrm{Co} / \mathrm{Pt}$ by optimizing the interface roughness. Indeed, recent experiments show that interface roughness is crucial for the helicity-dependent photocurrent [20].

Finally, we discuss the dependence of the magnetic photogalvanic effect on the SOI-strength $\alpha^{\mathrm{R}}$. The laserinduced charge-current density is shown as a function of $\alpha^{\mathrm{R}}$ in Fig. 3, where we set $\mathcal{E}_{\mathrm{F}}=1.36 \mathrm{eV}, \Delta V=1 \mathrm{eV}$, $\Gamma=25 \mathrm{meV}$ and $\hat{\boldsymbol{n}}$ points in $y$ direction. The magnetic photogalvanic effect increases strongly with the SOI strength $\alpha^{\mathrm{R}}$. Already for $\alpha^{\mathrm{R}}=200 \mathrm{meV} \AA$, which is only twice as large as the value of $\alpha^{\mathrm{R}}$ used in Fig. 1 and in Fig. 2, the current density $J_{x}$ reaches $45.7 \mathrm{~A} / \mathrm{m}$ for linearly polarized light with polarization vector along $y$ direction and the current density $J_{y}$ reaches $0.59 \mathrm{~A} / \mathrm{m}$ for circularly polarized light, i.e., both $J_{x}$ and $J_{y}$ are larger than the maximal values in Fig. 1 by one order of magnitude. The laser-induced currents increase by another order of magnitude when $\alpha^{\mathrm{R}}$ is increased further to $340 \mathrm{meV} \AA$, where $J_{x}=630 \mathrm{~A} / \mathrm{m}$ and $J_{y}=9.8 \mathrm{~A} / \mathrm{m}$.

The question therefore arises whether $\alpha^{\mathrm{R}}$ can reach $300 \mathrm{meV} \AA$ or more in magnetic bilayer systems, in which case the magnetic photogalvanic effect would be very strong and would allow us to increase the efficiency of table-top THz emitters [1] much further. While large Rashba SOI-strengths of $\alpha^{\mathrm{R}}=3.05 \mathrm{eV} \AA$ have been found in $\mathrm{Bi} / \mathrm{Ag}(111)$ surface alloys [27], the estimated values of 


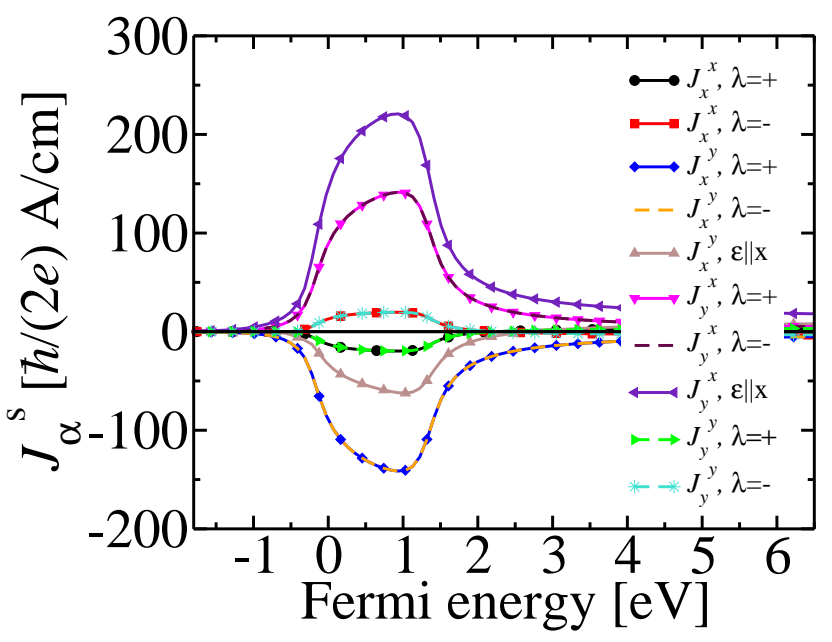

FIG. 4. Laser-induced spin-current density $J_{\alpha}^{s}$ vs. Fermi energy in the nonmagnetic Rashba model for the parameters $\alpha^{\mathrm{R}}=2 \mathrm{eVA}$ and $\Gamma=136 \mathrm{meV}$.

$\alpha^{\mathrm{R}}$ in magnetic bilayer systems are often smaller, e.g. $\alpha^{\mathrm{R}}=0.095 \mathrm{eV} \AA$ in $\mathrm{Co} / \mathrm{Pt}$ magnetic bilayer systems 34 . While one may expect that magnetic bilayer systems with larger $\alpha^{\mathrm{R}}$ will be discovered, it is likely that magnetic adatoms on surfaces or 2D materials are also systems with large magnetic photogalvanic effect. For example in graphene decorated with $\mathrm{W}$ and in semi-hydrogenated $\mathrm{Bi}(111)$ bilayers very large SOTs have been found in $a b$ initio calculations [39].

\section{B. Laser-induced spin currents}

Next, we discuss the laser-induced spin currents in the

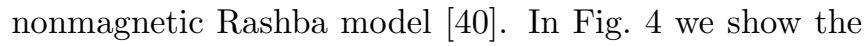
laser-induced spin current as a function of Fermi energy $\mathcal{E}_{\mathrm{F}}$ for the parameters $\alpha^{\mathrm{R}}=2 \mathrm{eV} \AA$ and $\Gamma=136 \mathrm{meV}$. Since one electron carries a spin angular momentum of $\hbar / 2$ it is convenient to discuss spin currents in units of $\hbar /(2 e)$ times ampere. Therefore, we use in Fig. 4 $\hbar /(2 e) \mathrm{A} / \mathrm{cm}$ as unit of the spin current density. A spin current of $1 \mathrm{~A} \hbar /(2 e)$ can be thought of as a positive charge current of $0.5 \mathrm{~A}$ carried by spin-down electrons accompanied by a negative charge current of $0.5 \mathrm{~A}$ carried by spin-up electrons. In agreement with the discussion in section IIIB summarized in Table III the following components are nonzero in the nonmagnetic case: For linearly polarized light only $J_{y}^{x}$ and $J_{x}^{y}$ are allowed by symmetry. For circularly polarized light $J_{y}^{x}$ and $J_{x}^{y}$ (even in the helicity $\lambda$ ) and $J_{y}^{y}$ and $J_{x}^{x}$ (odd in the helicity $\lambda$ ) are allowed by symmetry. Light linearly polarized along $x$ induces a smaller $J_{x}^{y}$ than circularly polarized light. However, light linearly polarized along $x$ induces a larger $J_{y}^{x}$ than circularly polarized light. For circularly polarized light rotation around the $z$ axis by $90^{\circ}$ is a symmetry operation, which leads to $J_{x}^{y}=-J_{y}^{x}$ and to $J_{x}^{x}=J_{y}^{y}$.

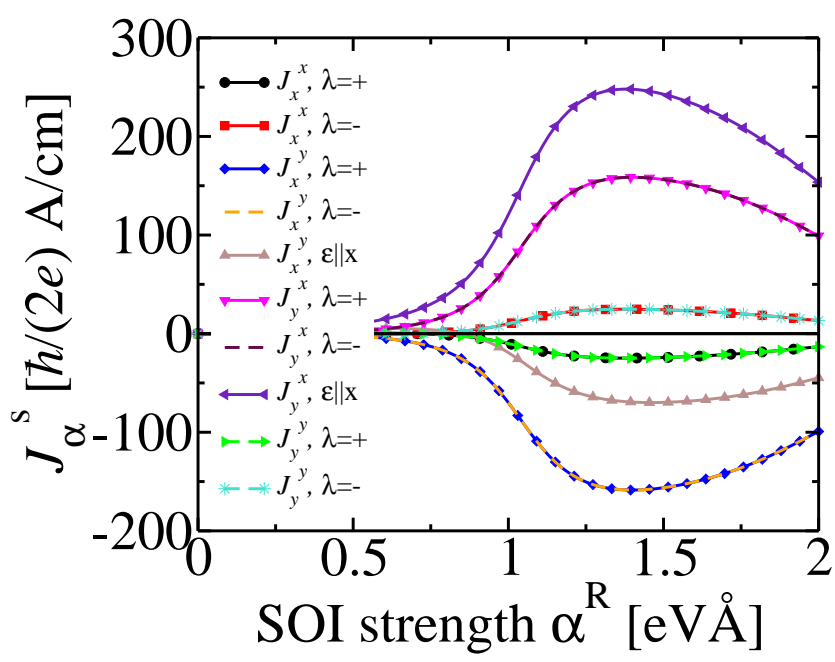

FIG. 5. Laser-induced spin-current density $J_{\alpha}^{s}$ vs. SOIstrength $\alpha^{\mathrm{R}}$ in the nonmagnetic Rashba model for the parameters $\mathcal{E}_{\mathrm{F}}=1.36 \mathrm{eV}$ and $\Gamma=136 \mathrm{meV}$.

For the parameter range covered by Fig. 4 the helicityodd effects are much smaller than the helicity-even effects. The maximum spin-current density in Fig. 4 is attained by the component $J_{y}^{x}$ for light polarized linearly along $x$ and it amounts to $J_{y}^{x}=221 \hbar /(2 e) \mathrm{A} / \mathrm{cm}$. This spin-current density can be thought of as a charge-current density of spin-up electrons (spin-up and spin-down refer to the $x$ axis as spin-quantization axis) of $110 \mathrm{~A} / \mathrm{cm}$ flowing into the negative $y$ direction and a charge-current density of spin-down electrons of $110 \mathrm{~A} / \mathrm{cm}$ flowing into the positive $y$ direction. This spin-dependent chargecurrent density of $\pm 110 \mathrm{~A} / \mathrm{cm}$ exceeds the laser-induced charge-current density that has been measured experimentally in magnetic bilayer systems [1, 3, 5] at comparable light intensity by several orders of magnitude. Since the net charge current is zero it does not generate a $\mathrm{THz}$ electromagnetic signal, which makes this effect difficult to observe experimentally. The inverse spin Hall effect could be used to convert these spin currents into detectable charge currents. However, this would require to inject the spin current from the Rashba system into a different system, because in the Rashba model itself there is no inverse spin Hall effect that converts any of the spin-current densities $J_{y}^{x}, J_{x}^{y}, J_{y}^{y}$ or $J_{x}^{x}$ into a charge current.

In Fig. 5 we show the laser-induced spin-current density as a function of SOI strength $\alpha^{\mathrm{R}}$ for the parameters $\mathcal{E}_{\mathrm{F}}=1.36 \mathrm{eV}$ and $\Gamma=136 \mathrm{meV}$. The figure shows that for SOI strengths larger than $\alpha^{\mathrm{R}}=1 \mathrm{eV} \AA$ the effect is particularly sizable. In Fig. 6 we show the laser-induced spin-current density as a function of SOI strength $\alpha^{\mathrm{R}}$ for the smaller broadening of $\Gamma=25 \mathrm{meV}$ at the Fermi energy $\mathcal{E}_{\mathrm{F}}=1.36 \mathrm{eV}$. For this smaller broadening much larger spin-current densities can be reached. 


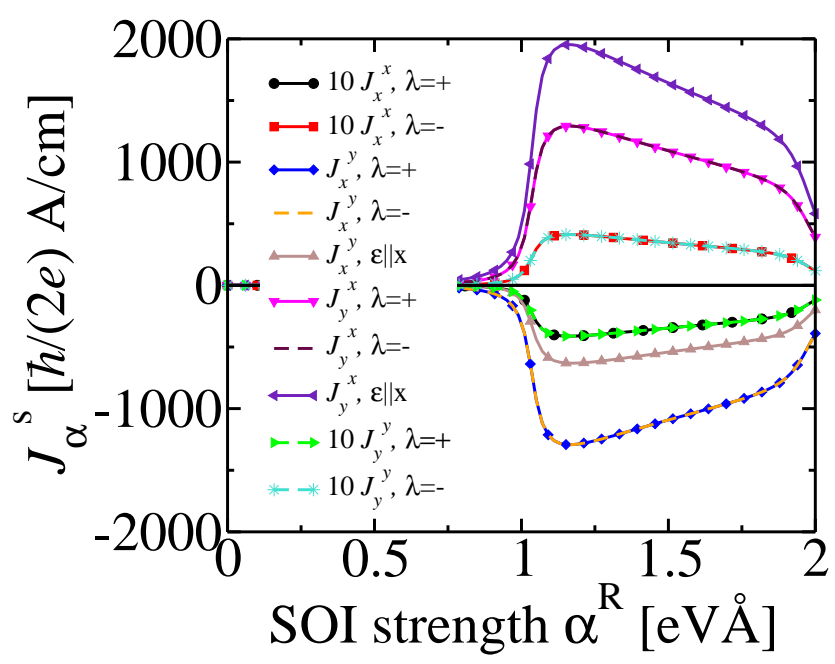

FIG. 6. Laser-induced spin-current density $J_{\alpha}^{s}$ vs. SOIstrength $\alpha^{\mathrm{R}}$ in the nonmagnetic Rashba model for the parameters $\mathcal{E}_{\mathrm{F}}=1.36 \mathrm{eV}$ and $\Gamma=25 \mathrm{meV}$. Some curves have been multiplied by the factor 10 for better visibility, as indicated in the legend.

\section{SUMMARY}

We study the laser-induced charge current in the ferromagnetic Rashba model with in-plane magnetization and predict that this magnetic photogalvanic effect is sufficiently strong in magnetic bilayer systems to be observable in experiments. The magnetic photogalvanic effect has one component that is odd in the helicity of light and a second component that is even in the helicity of light. The helicity-odd component can be maximized by optimizing the amount of disorder in the system. The helicity-even component depends strongly on the direction of the light-polarization vector when linearly polarized light is used. Additionally, we discuss laser-induced spin currents in the nonmagnetic Rashba model. Thereby, we predict that laser-induced pure spin currents at nonmagnetic surfaces and interfaces with giant Rashba effect exceed the laser-induced charge currents in magnetic bilayer systems such as $\mathrm{Co} / \mathrm{Pt}$ by several orders of magnitude.

\section{ACKNOWLEDGMENTS}

We acknowledge financial support from Leibniz Collaborative Excellence project OptiSPIN - Optical Control of Nanoscale Spin Textures, and funding under SPP 2137 "Skyrmionics" of the DFG. We gratefully acknowledge financial support from the European Research Council (ERC) under the European Union's Horizon 2020 research and innovation program (Grant No. 856538, project "3D MAGiC"), and ITN Network COMRAD. The work was also supported by the Deutsche Forschungsgemeinschaft (DFG, German Research Foundation) - TRR $173-268565370$ (project A11), TRR 288 - 422213477 (project B06). We also gratefully acknowledge the Jülich Supercomputing Centre and RWTH Aachen University for providing computational resources under project No. jiff40.
[1] T. Seifert, S. Jaiswal, U. Martens, J. Hannegan, L. Braun, P. Maldonado, F. Freimuth, A. Kronenberg, J. Henrizi, I. Radu, E. Beaurepaire, Y. Mokrousov, P. M. Oppeneer, M. Jourdan, G. Jakob, D. Turchinovich, L. M. Hayden, M. Wolf, M. Münzenberg, M. Kläui, and T. Kampfrath, Efficient metallic spintronic emitters of ultrabroadband terahertz radiation, Nature photonics 10, 483 (2016).

[2] E. T. Papaioannou and R. Beigang, Thz spintronic emitters: a review on achievements and future challenges, Nanophotonics , 20200563 (2020).

[3] T. Kampfrath, M. Battiato, P. Maldonado, G. Eilers, J. Nötzold, S. Mährlein, V. Zbarsky, F. Freimuth, Y. Mokrousov, S. Blügel, M. Wolf, I. Radu, P. M. Oppeneer, and M. Münzenberg, Terahertz spin current pulses controlled by magnetic heterostructures, Nature nanotechnology 8, 256 (2013).

[4] T. J. Huisman, C. Ciccarelli, A. Tsukamoto, R. V. Mikhaylovskiy, T. Rasing, and A. V. Kimel, Spinphoto-currents generated by femtosecond laser pulses in a ferrimagnetic $\mathrm{GdFeCo} / \mathrm{Pt}$ bilayer, Applied Physics Letters 110, 072402 (2017).

[5] T. J. Huisman, R. V. Mikhaylovskiy, J. D. Costa, F. Freimuth, E. Paz, J. Ventura, P. P. Freitas, S. Blügel,
Y. Mokrousov, T. Rasing, and A. V. Kimel, Femtosecond control of electric currents in metallic ferromagnetic heterostructures, Nature nanotechnology 11, 455 (2016)

[6] M. Battiato, K. Carva, and P. M. Oppeneer, Superdiffusive spin transport as a mechanism of ultrafast demagnetization, Phys. Rev. Lett. 105, 027203 (2010).

[7] M. Battiato, K. Carva, and P. M. Oppeneer, Theory of laser-induced ultrafast superdiffusive spin transport in layered heterostructures, Phys. Rev. B 86, 024404 (2012).

[8] G. Malinowski, F. Dalla Longa, J. H. H. Rietjens, P. V. Paluskar, R. Huijink, H. J. M. Swagten, and B. Koopmans, Control of speed and efficiency of ultrafast demagnetization by direct transfer of spin angular momentum, Nature physics 4, 855 (2008).

[9] A. Melnikov, I. Razdolski, T. O. Wehling, E. T. Papaioannou, V. Roddatis, P. Fumagalli, O. Aktsipetrov, A. I. Lichtenstein, and U. Bovensiepen, Ultrafast transport of laser-excited spin-polarized carriers in $\mathrm{Au} / \mathrm{Fe} / \mathrm{MgO}$ (001), Phys. Rev. Lett. 107, 076601 (2011).

[10] G.-M. Choi, A. Schleife, and D. G. Cahill, Opticalhelicity-driven magnetization dynamics in metallic ferromagnets, Nature Communications 8, 15085 (2017).

[11] F. Freimuth, S. Blügel, and Y. Mokrousov, Direct and inverse spin-orbit torques, Phys. Rev. B 92, 064415 (2015). 
[12] F. Freimuth, S. Blügel, and Y. Mokrousov, Charge pumping driven by the laser-induced dynamics of the exchange splitting, Phys. Rev. B 95, 094434 (2017).

[13] K. Krieger, P. Elliott, T. Müller, N. Singh, J. K. Dewhurst, E. K. U. Gross, and S. Sharma, Ultrafast demagnetization in bulk versus thin films: an ab initio study, Journal of Physics: Condensed Matter 29, 224001 (2017)

[14] W. Töws and G. M. Pastor, Many-body theory of ultrafast demagnetization and angular momentum transfer in ferromagnetic transition metals, Phys. Rev. Lett. 115, 217204 (2015).

[15] E. Carpene, H. Hedayat, F. Boschini, and C. Dallera, Ultrafast demagnetization of metals: Collapsed exchange versus collective excitations, Phys. Rev. B 91, 174414 (2015).

[16] S. Eich, M. Plötzing, M. Rollinger, S. Emmerich, R. Adam, C. Chen, H. C. Kapteyn, M. M. Murnane, L. Plucinski, D. Steil, B. Stadtmüller, M. Cinchetti, M. Aeschlimann, C. M. Schneider, and S. Mathias, Band structure evolution during the ultrafast ferromagnetic-paramagnetic phase transition in cobalt, Science Advances 3, e1602094 (2017).

[17] S. D. Ganichev and W. Prettl, Spin photocurrents in quantum wells, Journal of Physics: Condensed Matter 15, R935 (2003).

[18] Q. Ma, S.-Y. Xu, C.-K. Chan, C.-L. Zhang, G. Chang, Y. Lin, W. Xie, T. Palacios, H. Lin, S. Jia, P. A. Lee, P. Jarillo-Herrero, and N. Gedik, Direct optical detection of weyl fermion chirality in a topological semimetal, Nature Physics 13, 842 (2017).

[19] J. W. McIver, D. Hsieh, H. Steinberg, P. JarilloHerrero, and N. Gedik, Control over topological insulator photocurrents with light polarization, Nature Nanotechnology 7, 96 (2012).

[20] G. Li, R. Medapalli, R. V. Mikhaylovskiy, F. E. Spada, T. Rasing, E. E. Fullerton, and A. V. Kimel, Thz emission from $\mathrm{Co} / \mathrm{Pt}$ bilayers with varied roughness, crystal structure, and interface intermixing, Phys. Rev. Materials 3, 084415 (2019)

[21] A. Manchon, H. C. Koo, J. Nitta, S. M. Frolov, and R. A. Duine, New perspectives for Rashba spin-orbit coupling, Nature materials 14, 871 (2015).

[22] R. D. R. Bhat, F. Nastos, A. Najmaie, and J. E. Sipe, Pure spin current from one-photon absorption of linearly polarized light in noncentrosymmetric semiconductors, Phys. Rev. Lett. 94, 096603 (2005).

[23] H. Zhao, X. Pan, A. L. Smirl, R. D. R. Bhat, A. Najmaie, J. E. Sipe, and H. M. van Driel, Injection of ballistic pure spin currents in semiconductors by a single-color linearly polarized beam, Phys. Rev. B 72, 201302(R) (2005).

[24] S. D. Ganichev, V. V. Bel'kov, S. A. Tarasenko, S. N. Danilov, S. Giglberger, C. Hoffmann, E. L. Ivchenko, D. Weiss, W. Wegscheider, C. Gerl, D. Schuh, J. Stahl, J. De Boeck, G. Borghs, and W. Prettl, Zero-bias spin separation, NATURE PHYSICS 2, 609 (2006).

[25] J. Rioux and G. Burkard, Photoinduced pure spincurrent injection in graphene with rashba spin-orbit interaction, Phys. Rev. B 90, 035210 (2014)

[26] J. Li and P. M. Haney, Optical spintronics in organic-inorganic perovskite photovoltaics, Phys. Rev. B 93, 155432 (2016)

[27] C. R. Ast, J. Henk, A. Ernst, L. Moreschini, M. C. Falub, D. Pacilé, P. Bruno, K. Kern, and M. Grioni, Giant spin splitting through surface alloying,
Phys. Rev. Lett. 98, 186807 (2007).

[28] A. Crepaldi, L. Moreschini, G. Autès, C. TournierColletta, S. Moser, N. Virk, H. Berger, P. Bugnon, Y. J. Chang, K. Kern, A. Bostwick, E. Rotenberg, O. V. Yazyev, and M. Grioni, Giant ambipolar rashba effect in the semiconductor BiTeI, Phys. Rev. Lett. 109, 096803 (2012)

[29] K. Ishizaka, M. S. Bahramy, H. Murakawa, M. Sakano, T. Shimojima, T. Sonobe, K. Koizumi, S. Shin, H. Miyahara, A. Kimura, K. Miyamoto, T. Okuda, H. Namatame, M. Taniguchi, R. Arita, N. Nagaosa, K. Kobayashi, Y. Murakami, R. Kumai, Y. Kaneko, Y. Onose, and Y. Tokura, Giant rashba-type spin splitting in bulk BiTeI, NATURE MATERIALS 10, 521 (2011).

[30] M. Sakano, M. S. Bahramy, A. Katayama, T. Shimojima, H. Murakawa, Y. Kaneko, W. Malaeb, S. Shin, K. Ono, H. Kumigashira, R. Arita, N. Nagaosa, H. Y. Hwang, Y. Tokura, and K. Ishizaka, Strongly spin-orbit coupled two-dimensional electron gas emerging near the surface of polar semiconductors, Phys. Rev. Lett. 110, 107204 (2013)

[31] P. D. C. King, R. C. Hatch, M. Bianchi, R. Ovsyannikov, C. Lupulescu, G. Landolt, B. Slomski, J. H. Dil, D. Guan, J. L. Mi, E. D. L. Rienks, J. Fink, A. Lindblad, S. Svensson, S. Bao, G. Balakrishnan, B. B. Iversen, J. Osterwalder, W. Eberhardt, F. Baumberger, and $\mathrm{P}$. Hofmann, Large tunable rashba spin splitting of a two-dimensional electron gas in $\mathrm{Bi}_{2} \mathrm{Se}_{3}$, Phys. Rev. Lett. 107, 096802 (2011)

[32] V. V. Volobuev, P. S. Mandal, M. Galicka, O. Caha, J. Sanchez-Barriga, D. Di Sante, A. Varykhalov, A. Khiar, S. Picozzi, G. Bauer, P. Kacman, R. Buczko, O. Rader, and G. Springholz, Giant rashba splitting in $\mathrm{Pb}_{1-x} \mathrm{Sn}_{x} \mathrm{Te}(111)$ topological crystalline insulator films controlled by $\mathrm{Bi}$ doping in the bulk, Advanced Materials 29, 1604185 (2017).

[33] F. Freimuth, S. Blügel, and Y. Mokrousov, Laser-induced torques in metallic ferromagnets, Phys. Rev. B 94, 144432 (2016).

[34] K.-W. Kim, H.-W. Lee, K.-J. Lee, and M. D. Stiles, Chirality from interfacial spin-orbit coupling effects in magnetic bilayers, Phys. Rev. Lett. 111, 216601 (2013).

[35] A. J. Berger, E. R. J. Edwards, H. T. Nembach, A. D. Karenowska, M. Weiler, and T. J. Silva, Inductive detection of fieldlike and dampinglike ac inverse spin-orbit torques in ferromagnet/normal-metal bilayers, Phys. Rev. B 97, 094407 (2018)

[36] E. L. Ivchenko and S. D. Ganichev, Spin-photogalvanics, in Spin Physics in Semiconductors, edited by M. I. Dyakonov (Springer Berlin Heidelberg, Berlin, Heidelberg, 2008) pp. 245-277.

[37] F. Freimuth, S. Blügel, and Y. Mokrousov, Spin-orbit torques in $\mathrm{Co} / \mathrm{Pt}(111)$ and $\mathrm{Mn} / \mathrm{W}(001)$ magnetic bilayers from first principles, Phys. Rev. B 90, 174423 (2014).

[38] K. Garello, I. M. Miron, C. O. Avci, F. Freimuth, Y. Mokrousov, S. Blügel, S. Auffret, O. Boulle, G. Gaudin, and P. Gambardella, Symmetry and magnitude of spin-orbit torques in ferromagnetic heterostructures, Nature Nanotech. 8, 587 (2013).

[39] J.-P. Hanke, F. Freimuth, C. Niu, S. Blügel, and Y. Mokrousov, Mixed weyl semimetals and lowdissipation magnetization control in insulators by spinorbit torques, Nature Communications 8, 1479 (2017) 
[40] E. Y. Sherman, A. Najmaie, and J. E. Sipe, Spin current injection by intersubband transitions in quantum wells, Applied Physics Letters 86, 122103 (2005). 